\title{
Rhéologie des laves torrentielles
}

\author{
Philippe Coussot \\ CEMAGREF, Division Protection contre les Erosions, \\ Domaine Universitaire, B.P. 76, 38402 St-Martin-d'Hères, France \\ Tél. : 76762766
}

\begin{abstract}
Les laves torrentielles sont des volumes importants (de quelques $\mathrm{m}^{3}$ jusqu'à plusieurs millions de $\mathrm{m}^{3}$ ) d'un mélange d'eau, de matières organiques, d'argiles, de sable et de cailloux de toutes tailles (jusqu'à plusieurs $\mathrm{m}^{3} \mathrm{de}$ diamètre), avec une concentration solide très élevée (entre 50 et $85 \%$ en volume), et qui s'écoulent parfois dans les torrents de montagne après de longues ou fortes pluies. Parce qu'elles peuvent engendrer des dégâts importants, il apparaît nécessaire de maîtriser au mieux les techniques de prévention et de protection contre ces phénomènes (barrages, plages de dépôt, délimitation des zones à risque,...). Dans ce but la connaissance des lois d'écoulement de ces matériaux est une base essentielle. Comme il s'agit de fluides a priori non-newtoniens s'écoulant sur des fortes pentes il n'est pas envisageable d'utiliser les lois de l'hydraulique à surface libre d'eau claire. Le préalable indispensable à l'étude ou la description des mouvements de tels matériaux est la connaissance de leurs lois de comportement. Le but de cet article est de passer en revue les connaissances actuelles dans ce domaine. On se basera essentiellement sur les résultats de recherches développées récemment essentiellement au Laboratoire de Rhéologie (Grenoble), ainsi qu'au CEMAGREF et à l'étranger.
\end{abstract}

Dans une première partie on se propose d'examiner les lois de comportement des différents matériaux que l'on peut obtenir en mélangeant de l'eau, de l'argile et des grains de toutes tailles. Dans la seconde partie, on établit une classification des laves torrentielles en fonction de leur loi de comportement. Enfin dans la troisième partie on passe en revue les principales techniques de mesure pratiques des propriétés rhéologiques des laves torrentielles.

\section{Lois de comportement des mélanges eau-argiles- grains}

\subsection{Hypothèses préalables}

Les laves torrentielles sont des écoulements transitoires (sous formes de bouffées [1]) d'une masse d'eau, d'argiles, de sables et de cailloux de toutes tailles, qui roulent, glissent ou s'écoulent (cf. fig. I). Si on envisage tous les phénomènes particuliers qui peuvent se produire localement, la description de l'ensemble du phénomène s'avère très complexe. Cependant on peut simplifier le problème en faisant les hypothèses suivantes: dans une bouffée on trouve un front granulaire de faible volume à l'avant, puis le corps de lave d'un grand volume (cf. fig. l), enfin la

\section{Debris flow rheology}

Debris flows are large masses of water, clay, silt, sand, pebbles and boulders which sometimes flow in mountain streams after long or intense rains and can cause much damages to inhabited areas. We assume that these materials can be considered as homogeneous fluids. Usual hydraulic methods do not allow to predict natural flows of these non-Newtonian fluids. To make progress in this way the basic tool is the constitutive equation of the material.

In this paper we review our present knowledge in the field of rheological properties of the various possible water-debris mixtures. Thus we demonstrate that it is possible to distinguish two main debris flow types: muddy debris flows, which contain at least a moderate clay fraction (>10\%) and exhibit simple shear behaviour which may be fitted by a Herschel-Bulkley model; granular debris flows, which contain a very low clay fraction $(<1 \%)$ and exhibit a simple shear flow curve with a minimum. All these materials exhibit a yield stress (flow threshold) which is a key rheological parameter. We review the main practical techniques to estimate this yield stress. 
queue de lave de faible viscosité et que l'on négligera parce que son impact est faible. Les deux parties principales (front et corps) peuvent être considérées à une échelle d'observation globale comme des fluides homogènes se déformant continûment. Dans cette première approche, le glissement aux parois, la fracturation, la sédimentation, la ségrégation, l'érosion, les dépôts, etc, seront négligés. L'importance de ces différents phénomènes sur les écoulements naturels devra être examinée ultérieurement. Cependant, le fait de les négliger permettra d'estimer les caractéristiques des écoulements de manière relativement simple.

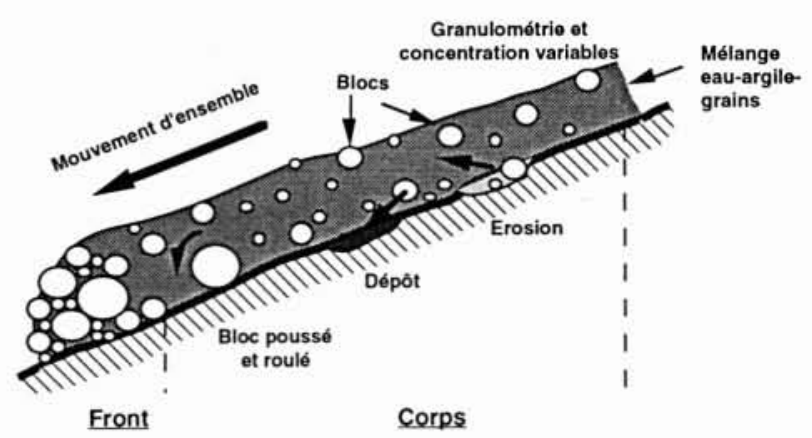

1. Profil de la structure interne d'une lave torrentielle.

\subsection{Lois de comportement des suspensions naturelles concentrées}

On peut voir le matériau constitutif (front ou corps) d'une lave torrentielle comme un certain volume d'eau dans lequel on ajoute progressivement des particules solides de plus en plus grosses (des argiles jusqu'aux blocs). En suivant cette idée il est possible de passer en revue et comprendre d'un point de vue microstructurel les différents types de lois de comportement des suspensions naturelles concentrées. Dans la suite, on étudie uniquement des mélanges dont la concentration solide est telle que le matériau ne se fracture ni ne sédimente pendant la plupart des écoulements naturels. En outre on ne s'intéresse pas aux écoulements très rapides pour lesquels la turbulence ou les transferts de quantité de mouvement par le biais de chocs inter-granulaires peuvent devenir prédominants. Le lecteur souhaitant approfondir d'un point de vue général la notion de loi de comportement pourra utilement consulter les références [2-5].

\subsubsection{Les suspensions boueuses fines}

Par la procédure consistant à ajouter successivement des particules de plus en plus grosses, on obtient d'abord un mélange eau-argile. A des concentrations solides pas trop élevées ce type de fluide est vraisemblablement celui que l'on trouve dans les écoulements hyperconcentrés [6]. Les mélanges eau-argile purifiée peuvent avoir des propriétés temporelles marquées [7] mais la loi de comportement des mélanges eau-argile naturelle saturés en cations suit en général [8-10] un modèle de Herschel \& Bulkley, qui, en cisaillement simple, s'exprime ainsi :

$\tau=\tau_{c}+K \dot{\gamma}^{n}$ quand $\dot{\gamma} \neq 0$, et $\tau \leqslant \tau_{c}$ quand $\dot{\gamma}=0$

où $\tau$ est la contrainte tangentielle, $\dot{\gamma}$ le gradient de vitesse, et $\tau_{c}, K$, et $n$ sont des paramètres du fluide. $\tau_{c}$ est le seuil de contrainte du fluide et représente la contrainte minimum à dépasser pour initier un écoulement. Le modèle de Herschel-Bulkley est un modèle purement empirique qui doit être calé sur les mesures obtenues en régime permanent avec un rhéomètre (cf. fig. 2). Dans de nombreux travaux antéricurs [11-13] le paramètre $n$ a été supposé égal à 1 , ce qui correspond à un modèle de Bingham. Cependant pour prédire correctement le plus grand éventail possible d'ćcoulements à l'äide d'un tel modèle mathématique il est recommandé de caler ce modèle sur les mesures dans la plus large gamme possible de gradient de vitesse. Pour cela il apparaît préférable d'utiliser une valeur de $n$ proche de $\frac{1}{3}[10,14]$. Il est possible de caler différents triplets de paramètres proches de facon correcte sur les données expérimentales mais cela n'affecte pas la valeur finale du seuil de contrainte de facon significative. Il est important de noter qu'en calant ce modèle sur une gamme incluant de faibles valeurs de gradients de vitesse on obtient une valeur du seuil qui est très proche du seuil réel du matériau [10]. Si en revanche on cale un modèle de Bingham sur une gamme restreinte de gradients de vitesse on commet une erreur qui peut dépasser $100 \%$ sur la valeur du seuil (cf. fig. 2).

Les trois paramètres du modèle de Herschel-Bulkley ne varient pas sensiblement avec le $\mathrm{pH}$, la température, ou la

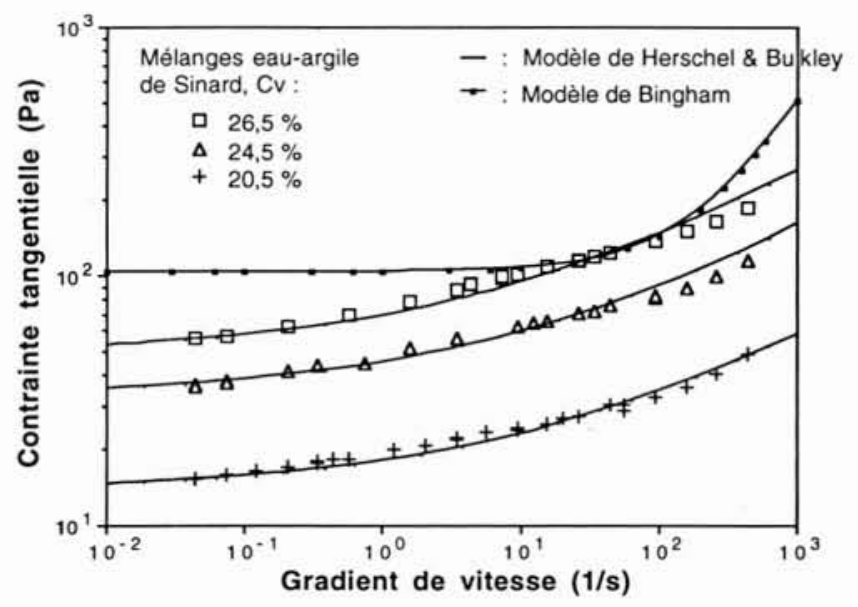

2. Loi de comportement en cisaillement simple d'un mèlange canargile à diffërentes concentrations solides. Calage d'un modèle de Herschel-Bulkley. Dans le cas du mélange le plus concentré. calage d'un modèle de Bingham dans la gamme 10 à $100 \mathrm{~s}^{-1}[30]$. 
concentration en électrolyte (dans des gammes de variation correspondant aux conditions naturelles) $[10,15] . K$ et $\tau_{c}$ sont très sensibles à la concentration solide $\left(C_{v}\right)$ et on peut notamment écrire $[9-10,12]$ :

$$
\tau_{c}=a \exp \left(k C_{v}\right) ; K=a^{\prime} \exp \left(k^{\prime} C_{v}\right)
$$

où $a, a^{\prime}$ et $k, k^{\prime}$ sont des paramètres du fluide. $k$ et $k^{\prime}$ ont des valeurs généralement comprises entre 10 et 30 (avec $C_{v}$ exprimé en pourcentage). A priori ces paramètres diffèrent d'un matériau à l'autre.

\subsubsection{Laves torrentielles boueuses}

Si la fraction argileuse initiale dans un mélange eau-argile est suffisamment faible, l'effet, sur le seuil de contrainte, de l'addition de grains dans ce mélange, est dans certains cas négligeable $[14,16-17]$ tant que la concentration de grains ajoutée est inférieure à 10. Cependant en général, le seuil de contrainte de la suspension augmente rapidement avec la concentration solide totale lorsqu'on ajoute des grains [16]. Ceci peut s'exprimer de la manière suivante :

$$
\tau_{c}=A \exp \left(\chi C_{v}\right)
$$

où $A$ et $\chi$ sont des paramètres du fluide qui sont d'autant plus petits que la fraction de grains ajoutés a une granulométrie étendue. Les laves torrentielles ayant une granulométrie très étendue $[1,14]$, les constatations ci-dessus expliquent le fait que leur concentration solide totale est très élevée sans pour autant que leur seuil de contrainte soit trop grand. En outre tant que la fraction de grains ajoutés n'est pas trop grande (cf. 1.2.3), la loi de comportement des suspensions correspondantes reste formellement du type donné par l'équation (1) [18]. Ce résultat s'explique assez bien si on admet que le mélange eau-argiles interstitiel lubrifie les mouvements relatifs des grains (cf. fig. 2). L'ensemble des matériaux pour lesquels ces résultats s'appliquent seront appelés dans la suite «mélanges essentiellement boueux ".

\subsubsection{Laves torrentielles gramulaires}

Lorsqu'on continue à ajouter des grains dans un mélange eau-argiles comme précédemment on finit par obtenir un mélange qui a un seuil très élevé et se fracture facilement. Il est probable qu'un réseau très serré de contacts directs entre les grains (cf. fig. 3) du matériau n'autorise pas de grandes déformations continues. On ne peut plus considérer ce type de matériau comme un fluide.

$\mathrm{Si}$ en revanche la concentration argileuse dans ce type de mélange est très faible $(<1 \%)$, les éventuels contacts lubrifiés entre grains se font grâce à un fluide interstitiel très peu visqueux. Alors, il est possible qu'au cours du mouvement la configuration (l'arrangement) des particules évolue, en se dilatant ou non. Le nombre de contacts directs donnant lieu à des frictions va décroitre au profit du nombre de contacts lubrifiés qui dissipent moins d'énergie (au moins à des vitesses pas trop élevées) $[14,18]$. Une certaine forme d'écoulement pourra alors se produire. Le comportement global est "instable» au sens où lorsqu'on augmente

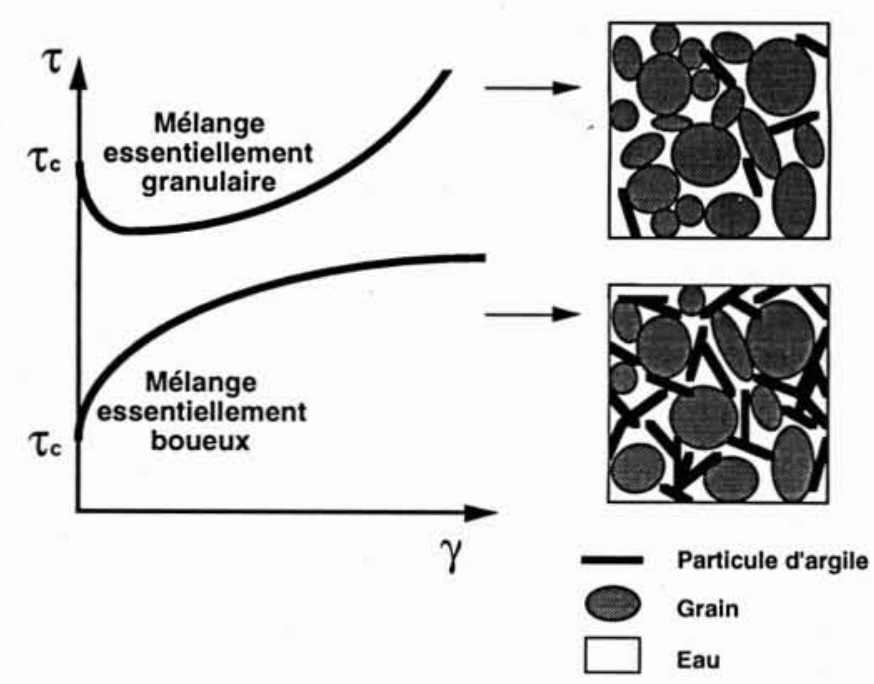

3. Principaux types de loi de comportement en cisaillement simple des mélanges eau-argiles-grains. Schémas de principe de la structure interne des matériaux correspondants.

progressivement la contrainte appliquée à ce type de matériau on observe la transition soudaine entre le repos et un cisaillement assez rapide au moment où la contrainte dépasse de peu le seuil de contrainte [19-20]. Ce type de comportement peut être partiellement représenté par une courbe d'écoulement en cisaillement simple ayant un minimum (cf. fig. 3). Malheureusement cette courbe est difficile à déterminer expérimentalement parce que des effets perturbateurs se produisent lors d'expériences de rhéométrie. On peut au moins conclure que, dans la nature, des "mélanges granulaires» de ce type sont susceptibles de s'arrêter ou de s'écouler à nouveau assez rapidement pour des faibles modifications des conditions aux limites (pente, section du canal).

Ce type de matériau semble correspondre à celui pour lequel TAKAHASH [21] a proposé un modèle de loi de comportement basé sur le travail de BAGNOLD [22] concernant des écoulements rapides de suspensions concentrées de grains identiques dans un fluide newtonien. BAGNOLD a distingué un régime macrovisqueux (faibles gradients de vitesse) pour lequel les dissipations visqueuses sont prédominantes, d'un régime inertiel (gradients de vitesse élevés) pour lequel les transferts de quantité de mouvement par chocs entre particules sont prédominants. Malheureusement il n'est pas possible d'appliquer a priori ces résultats aux écoulements de suspensions naturelles car ces dernières sont très concentrées et contiennent une granulométrie très étendue de particules solides. Notamment une faiblesse notable du modèle de Takahashi est qu'il n'inclut pas dans une seule loi de comportement l'existence d'un seuil de contrainte (qui explique notamment l'arrêt possible de faibles épaisseurs de laves sur des pentes relativement fortes). Cependant il n'est pas impossible que ce modèle permette de décrire correctement les écoulements très rapides de mélanges eau-grains sur des fortes pentes 
mais ceci n'a pas encore fait l'objet d'une démonstration nette. Bien au contraire il semble qu'on ne puisse pas décrire la loi de comportement d'un matériau granulaire sec en écoulement à surface libre à l'aide d'un modèle reliant tenseur des contraintes et tenseur des taux de déformations [23]. Quoi qu'il en soit, des progrès notoires restent à accomplir dans le domaine des lois de comportement des mélanges essentiellement granulaires.

\section{Classification des laves torrentielles en fonction de leur loi de comportement}

La difficulté qui se pose d'emblée pour établir une classification suffisamment précise des laves torrentielles tient dans la délimitation nette du domaine qui nous intéresse. Comme le montre Meunier [24], on peut considérer que du point de vue du matériau il existe un passage continu (du point de vue des composants) des écoulements hyperconcentrés aux laves torrentielles et des laves torrentielles au glissement de terrain. De manière à respecter nos hypothèses de départ (cf. 1.1) nous considèrerons qu'un écoulement est une lave torrentielle lorsque globalement on a affaire à un fluide homogène. Dans ces conditions le transport solide ou le charriage hyperconcentré se distingueront des laves torrentielles par le fait que lors de tels écoulements, les particules grossières ont une vitesse moyenne différente de celle du fluide interstitiel. De même les glissements de terrain se distingueront des laves torrentielles par le fait que les localisations des déformations deviennent prédominantes dès que le mouvement s'accélère. Ceci est en accord avec le fait que les mẻlanges qui sédimentent très rapidement ou se fracturent ont été écartés d'emblée dans notre discussion (cf. 1.2). S'il s'agit d'une suspension fine la distinction est évidemment plus difficile. Dans ce cas, on propose de considérer, de facon arbitraire, qu'il s'agit d'une lave torrentielle si le matériau est suffisamment visqueux pour s'écouler en régime laminaire dans la plupart des conditions naturelles.

Au cours du chapitre précédent, on a montré que la fraction argileuse présente dans un mélange joue un rôle clé pour le comportement de l'ensemble. Le mélange eauargiles lubrifie les contacts inter-granulaires. Dans ces conditions, un premier paramètre pertinent pour une classification rhéologique est la fraction d'argiles par rapport à la quantité totale de solide (cette fraction, exprimée en pourcentage, se rapporte aussi bien à des volumes qu'à des masses si on suppose que tous les matériaux solides ont la même densité). Par ailleurs compte-tenu des évolutions simples du seuil de contrainte des mélanges en fonction de la concentration solide (volumique), cette dernière quantité, exprimée en pourcentage, est évidemment le second paramètre pertinent pour décrire un mélange naturel du point de vue de sa loi de comportement. Tenir compte de la granulométrie serait tout à fait utile pour affiner les

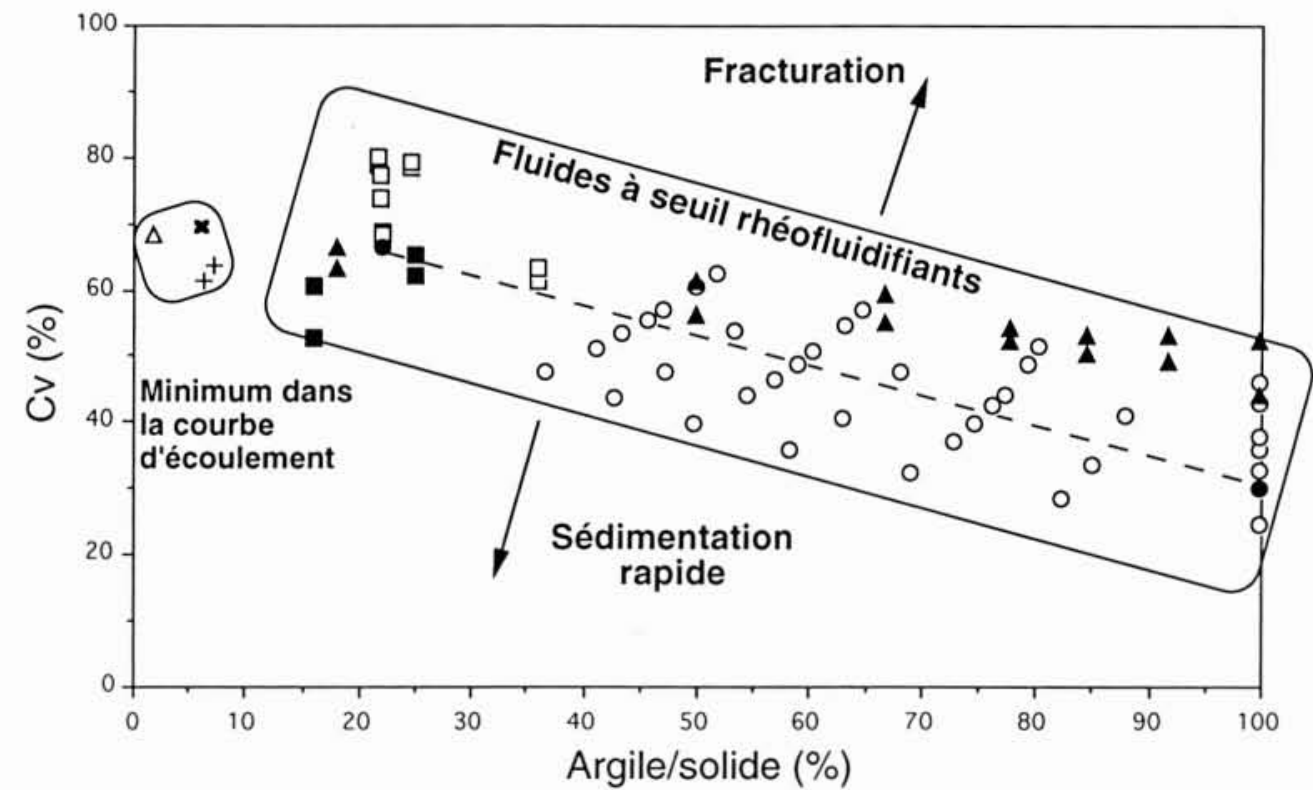

- Fraction fine de lave torrentielle $(<0,04 \mathrm{~mm})+$ sable $(0,1-0,2 \mathrm{~mm})$ [14]

口 Fraction de lave torrentielle $<20 \mathrm{~mm}$ [18]

$\triangle$ Fraction de lave torrentielle $<20 \mathrm{~mm}$ [18]

+ eau-argile + billes de polystyrene (0,1-0,2 mm) [14]

+ Fraction de lave torrentielle $<120 \mathrm{~mm}$ [28]

Modèle de

- Fraction de lave torrentielle $<35 \mathrm{~mm}$ [28]

- Fraction fine de lave torrentielle $(<0,074 \mathrm{~mm})+$ sable [12]

A Fraction fine de lave torrentielle $(<0,074 \mathrm{~mm})+$ sable [9]
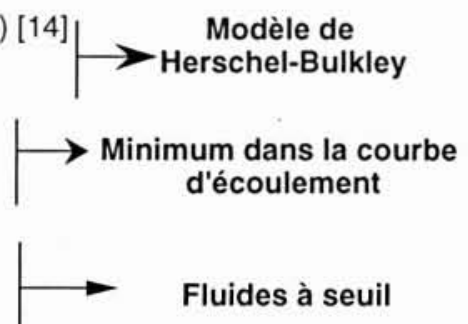

4. Loi de comportement en cisaillement simple des différents mélanges naturels testés dans la littérature en fonction de leur fraction argileuse et de leur concentration solide. 
descriptions qui vont suivre mais cela nous conduirait à une analyse trop complexe.

On présente figure 4 un diagramme (fraction argileuse, concentration solide) dans lequel on a reporté l'ensemble des résultats obtenus par les chercheurs dans le domaine des laves torrentielles qui ont testé des mélanges naturels relativement grossiers à l'aide de rhéomètres de grande taille. On voit apparaître clairement une zone (à droite) relative à des matériaux qui de manière générale suivent une loi de comportement du type Herschel-Bulkley (même si dans certains cas les auteurs des mesures ont plus simplement calé un modèle de Bingham sur les mesures obtenues). Cette zone, qui correspond finalement aux mélanges essentiellement boueux décrits au paragraphe précédent, se prolonge jusqu'à des fractions argileuses de l'ordre de $10 \%$. On propose d'appeler les écoulements naturels mettant en jeu des matériaux de ce type des «laves torrentielles essentiellement boueuses ». Une autre zone qui se distingue aussi clairement (à gauche) regroupe les mélanges qui ont une loi de comportement possédant un minimum. On propose d'appeler les écoulements mettant en jeu ce type de matériau des "laves torrentielles essentiellement granulaires». Bien que des expériences de rhéométrie soient difficilement envisageables sur les matériaux constituant le front des laves torrentielles, il est vraisemblable, étant donné la très forte concentration de gros blocs qu'il contient en général, que le front possède un comportement du type "mélange granulaire». On notera que les matériaux situés entre ces deux zones sont des matériaux intermédiaires qu'il est difficile de considérer comme des fluides dans les conditions usuelles parce qu'ils se fracturent ou sédimentent rapidement. Les matériaux situés en-dessous de ces zones correspondent à des mélanges constitutifs des écoulements avec transport solide. Les matériaux situés au-dessus de ces zones correspondent à des roches ou des sols saturés d'eau.

\section{Détermination des paramètres rhéologiques}

Les rhéomètres de laboratoire permettent de réaliser des tests sur des petits volumes de fluide (ordre de grandeur: $1 \mathrm{~cm}^{3}$ ) et un échantillon complet de lave torrentielle ne peut évidemment pas être étudié par ce moyen. Il est cependant possible, avec ces rhéomètres, de tester le mélange eau-argile interstitiel des laves. Mais même dans ce cas relativement simple les expériences doivent être réalisées avec beaucoup de précautions pour obtenir des résultats qui représentent effectivement la loi de comportement du fluide $[7,10,14,25]$. Les mélanges qui nous concernent font en effet partie de la grande classe des fluides à seuil qui posent de nombreux problèmes expérimentaux [26-27] et font encore l'objet de quelques débats conceptuels dans le domaine de la rhéologie. Par ailleurs quelques rhéomètres de grande taille ont été construits dans le monde dans le but de tester des mélanges plus grossiers, mais, bien qu'ils aient déjà fourni quelques résultats intéressants, leur utilisation représente encore une opération lourde, coûteuse et complexe [9, 18, 28]. Enfin diverses méthodes de détermination approximative des caractéristiques rhéologiques d'un fluide existent mais doivent encore faire leurs preuves dans le domaine des fluides à seuil [29]. Quelle que soit la méthode de mesure utilisée il est nécessaire d'extrapoler (en utilisant éventuellement les courbes du type (3)) au mélange naturel les résultats obtenus sur des mélanges plus ou moins fins.

En pratique, de facon plus rapide mais moins précise, en admettant que les laves torrentielles boueuses ont toujours une loi de comportement du type Herschel-Bulkley, on peut estimer leur seuil de contrainte à partir de mesures simples. On utilise pour cela le résultat suivant tiré de la théorie des écoulements de fluides de Herschel-Bulkley : si on place une épaisseur uniforme $(h)$ de lave sur un plan infiniment large et long, le seuil de contrainte du fluide est donné par la formule suivante [30-31]:

$$
\tau_{c}=\rho g h(\sin i)
$$

où $\rho$ est la masse volumique du matériau, $g$ la pesanteur et $\mathrm{i}$ la pente critique (du canal) pour laquelle le fluide commence à couler (très lentement). Avec un canal de section arbitraire on peut utiliser l'approximation suivante :

$$
\tau_{c}=\rho g R(\sin i)
$$

où $R$ est le rayon hydraulique de l'écoulement. Pour connaître les domaines de validité de cette formule en fonction du type de section on se réfèrera à [32]. Pour ce qui est des autres paramètres de la loi de comportement on pourra prendre : $n=1 / 3$ et $K=\tau_{c} / 3$. Cette technique peut par exemple être appliquée, en laboratoire ou sur le terrain, à la matrice moyennement fine des laves $(<5$ ou $10 \mathrm{~cm})$. Le résultat obtenu pourra aussi être comparé à celui obtenu en effectuant le même type de mesure sur des dépôts naturels issus de l'arrêt progressif d'une lave en l'absence d'obstacle dans un canal de section uniforme. Cependant les conditions naturelles reproduisent rarement parfaitement les conditions théoriques nécessaires à l'utilisation de ces formules (inertie, obstacle, canal non uniforme,...). Cette dernière technique fournit donc généralement une approximation très grossière du seuil de contrainte de la lave naturelle.

Ces techniques peuvent aussi être utilisées dans le cas d'une lave granulaire mais l'incertitude sur la mesure du seuil de contrainte est encore plus grande. En effet, lorsque ce type de lave s'arrête, la contrainte à la paroi peut être très différente de la contrainte limite correspondant au début du mouvement (à partir du repos à contrainte imposée) (cf. fig. 2).

\section{Conclusion}

En utilisant les résultats acquis récemment en matière de rhéologie des mélanges naturels il nous a été possible de dresser une classification des laves torrentielles en fonction de leur loi de comportement. On a ainsi distingué deux types principaux : les laves torrentielles "essentiellement boueuses" dont la loi de comportement est du type Herschel-Bulkley, et les laves torrentielles « essentiellement granulaires » dont la loi de comportement peut être repré- 
sentée par une courbe avec un minimum. Cependant ce second type de mélange doit faire l'objet d'études plus approfondies. De manière plus générale le tableau simpliste dressé ici ne doit pas faire oublier la complexité de la réalité et des phénomènes naturels. Des études futures préciseront et complèteront ce tableau. Globalement ces résultats fournissent néanmoins une description et des méthodes de quantification des lois de comportement des laves torrentielles boueuses suffisante en première approximation pour passer à la phase suivante: l'étude des lois d'écoulement.

\section{Références}

[1] Davies T.R.H. (1986). - Large Debris Flows: A MacroViscous Phenomenon. Acta Mechanica, 63. pp. 161-178.

[2] Rhéologie et hydrotechnique (1970). — La Houille Blanche, $\mathrm{N}^{\circ} 5$.

[3] Coleman B.D., Markowitz H. and Noll W. (1966). Viscometric Flows of Non-Newtonian Fluids, Springer Tracts in Natural Philosophy. Volume 5, Springer Verlag BerlinHeidelberg-New-York, $130 \mathrm{p}$.

[4] PIAU J.M. (1979). - Fluides non-newtoniens. Techniques de l'Ingénieur, A 710-711.

[5] Coussot P. et Piau J.M. (1993), - L'écoulement des boues. La Recherche, 24, N*258, pp. 1084-1091.

[6] MEUNIER M. (1991). - Eléments d'hydraulique torrentielle. CEMAGreF, Collection Etudes, Série Montagne $N^{\circ} 1$, 274 p.

[7] Coussot P., Leonov A.I. and Piau J.-M. (1993). - Rheology of concentrated dispersed systems in a low molecular weight matrix. Journal of Non-Newtonian Fluid Mechanics, 46, pp. 179-217.

[8] Locat J. and Demers D. (1988). - Viscosity, yield stress, remolded strength, and liquidity index relationships for sensitive clays. Canadian Geotechnical Journal, 25, N4, pp. 799-806.

[9] MAJOR J.J. and PIERSON T.C. (1992). - Debris flow rheology : Experimental analysis of fine-grained slurries. Water Resources Research, 28, $\mathrm{N}^{*} 3$, pp. 841-857.

10] Coussot P. and PIAU J.-M. (1993). - On the behaviour of fine mud suspensions, soumis à Rheologica Acta.

[11] Johnson A.M. and Rodine J.R. (1984). - Debris flow, in Slope Instability (Editors David Brunsden and D.B. Prior), Chapter 8, John Wiley and Sons, New-York.

[12] O'BRIEN J.S., JULIEN P.Y. (1988). - Laboratory analysis of mudflows properties. Journal of Hydraulic Engineering, 114 (8), pp. 877-887.

[13] QIAN NING and WAN ZHAOHUI (1986). - A critical review of the research on the hyperconcentrated flow in China. International Research and Training Centre on Erosion and Sedimentation publication, China.

[14] Coussot P. (1992). - Rhéologie des laves torrentielles. Thése de Doctorat de l'Institut National Polytechnique de Grenoble, Grenoble, France, $420 \mathrm{p}$.
[15] Chang S.H., Ryan M.E. and Gupta R.K. (1993). - The effect of $\mathrm{pH}$, ionic strength, and temperature on the rheology and stability of aqueous clay suspensions. Rheologica Acta, 32 , pp. 263-269.

[16] Coussot P. and PIAU J.-M. (1993), - Effect of an addition of force-free particles on mud suspensions yield stress, en préparation pour Journal of Hydraulic Engineering.

[17] Migniot C. (1989). - Tassement et rhéologie des vases. La Houille Blanche, $\mathrm{N}^{\circ} 1$ et 2 .

[18] Coussot P. and PIAU J.-M. (1993) (c). - A large-scale field coaxial cylinder rheometer to study the rheology of natural coarse suspensions, soumis au Journal of Rheology.

[19] Jaeger H.M., Chu-Heng Liu, Nagel S.R. and Witten T.A. (1990). - Friction in granular flows. Europhysics Letters, 11 (7), pp. 619-624.

[20] KytomaA H.K. and Prasad D. (1993). - Transition from quasi-static to rate dependent shearing of concentrated suspensions. Powders \& Grains, Thornton (ed.), Balkema, Rotterdam.

[21] Tаканаshi T. (1981). - Debris flow. Annual Review of Fluid Mechanics, 13, pp. 57-77.

[22] BAGNOLD R.A. (1954). - Experiments on a gravity free dispersion of large solid spheres in a Newtonian fluid under shear. Proceedings of the Royal Society of London, A.225, pp. 49-63.

[23] ANCEY C. (1993). - Lois d'écoulement des matériaux granulaires. DEA Mécanique, INPG, Grenoble, France.

[24] Meunier M. (1994). - Les progrès de la connaissance et les méthodes d'étude des phénomènes torrentiels. La Houille Blanche (ce numéro).

[25] Coussot P. and Piau J.-M. (1993). - Techniques de rhéométrie en cisaillement simple dans le cas de dispersions et suspensions concentrées, sous presse dans les Cahiers de rhéologie, revue du Groupe Francais de Rhéologie.

[26] Magnin A., Piau J.M. (1987). - Shear rheometry of fluids with a yield stress. Journal of Non-Newtonian Fluid Mechanics, 23, pp. 91-106.

[27] MAGNIN A. and PIAU J.M. (1990). - Cone-and-plate rheometry of yield stress fluids. Study of an aqueous gel. Journal of Non-Newtonian Fluid Mechanics, 36, pp. 85-108.

[28] Phillips C.J. and Davies T.R.H. (1991). - Determining rheological parameters of debris flow material. Geomorphology, 4, pp. 101-110.

[29] NGuYen Q.D. and BOGER, D.V. (1992). - Measuring the flow properties of yield stress fluids. Annual Review of Fluid Mechanics, 24, pp. 47-88.

[30] Coussot P. (1993). - Steady, laminar, flow of concentrated mud suspensions in open channel, soumis au Journal of Hydraulic Research.

[31] Coussot P. (1993). - Méthode d'estimation pratique des paramètres rhéologiques des laves torrentielles boueuses. Note Technique, CEMAGREF, Division Protection contre les Erosions.

[32] Coussot P. (1994), - Lois d'écoulement des laves torrentielles. La Houille Blanche (ce numéro). 